\title{
MSSM scenarios with a light CP-odd Higgs boson
}

\author{
Mikhail Dubinin ${ }^{1,2, *}$ and Elena Fedotova ${ }^{2, * *}$ \\ ${ }^{1}$ Physics Department, Lomonosov Moscow State University, 119991 Moscow, Russia \\ ${ }^{2}$ Skobeltsyn Institute of Nuclear Physics, Lomonosov Moscow State University, 119991 Moscow, Rus- \\ sia
}

\begin{abstract}
The possibility of the presence of a light pseudoscalar with the mass of $28 \mathrm{GeV}$ in the MSSM Higgs sector extended by dimension-six operators is analyzed. The perturbative unitarity and vacuum stability constraints are discussed. Tree-level production cross sections of a light pseudoscalar in the process $g g \rightarrow \mu \mu b \bar{b}$, and cross sections of a light pseudoscalar production in the top quark decay are evaluated for suitable benchmark points.
\end{abstract}

\section{Introduction}

Light scalars are predicted in many extensions of the Standard Model (BSM) [1-4]. In light of these predictions an excess recently observed by CMS Collaboration in $p p \rightarrow b j \mu^{+} \mu^{-}$channel at $\sqrt{s}=8$ and $13 \mathrm{TeV}$ at the dimuon invariant mass $m_{\mu \mu} \approx 28 \mathrm{GeV}$ in two event categories (with $b$-jet in forward region and without $b$-jet) [5] is of interest. The local significances are not large: $4.2 \sigma$ and $2.9 \sigma$ at $\sqrt{s}=8 \mathrm{TeV}$ and $2.0 \sigma$ and $1.4 \sigma$ (deficit) at $\sqrt{s}=13 \mathrm{TeV}$. A similar search for a resonance decaying to two muons in the invariant mass range between 26 and $30 \mathrm{GeV}$ produced in association with a $b$-jet and additional jet performed by ATLAS experiment yields no evidence of new signal [6]. However, the comparison of ATLAS and CMS reconstruction procedures is very difficult due to differences in the $\mu$-trigger efficiency and $b$-tagging performance which suppress the signal/background ratio in the case of ATLAS and can be critical for such a weak signal. The situation is more intriguing in the light of a reanalysis of results of the ALEPH experiment at LEP where the excess has been found in the opposite-sign dimuon invariant mass spectra at $m_{\mu \mu}=30.40 \pm 0.46 \mathrm{GeV}$ in events containing $b$-quarks with a local significance $5 \sigma$ and a global significance $2.6 \sigma$ [7].

In the most popular extension of the Standard Model (SM) - the Minimal Supersymmetric Standard Model (MSSM) - such a signal could be identified with a light pseudoscalar Higgs boson, but such possibility is closed in commonly used approximations. The MSSM extended by dimension-six operators in the Higgs sector [8], however, allows such a possibility [9]. Note that this excess can be also explained in the framework of the Next-to-Minimal Supersymmetric Standard Model (NMSSM) [10] and can be treated in a study of the muon anomalous magnetic moment [11].

In this proceeding MSSM scenarios under the assumption that a CP-odd Higgs boson has a mass of about $28 \mathrm{GeV}$ are analyzed. Hereafter, an observed Higgs boson with a mass of $125 \mathrm{GeV}[12-15]$ is identified with a light CP-even scalar ${ }^{1}$.

\footnotetext{
*e-mail: dubinin@theory.sinp.msu.ru

**e-mail: fedotova@theory.sinp.msu.ru

${ }^{1}$ The possibility of SM-like heavy Higgs boson $H$ investigated in the case of nonzero $\kappa_{i}$ is excluded, see [9].
} 


\section{Higgs sector with dimension-six operators}

The MSSM Higgs potential at the low energy scale is an effective two-Higgs doublet model (THDM) potential which in general can be expanded to all orders of perturbation theory

$$
\begin{gathered}
U_{\mathrm{eff}}=U^{(2)}+U^{(4)}+U^{(6)}+\ldots, \\
U^{(2)}=-\mu_{1}^{2}\left(\Phi_{1}^{\dagger} \Phi_{1}\right)-\mu_{2}^{2}\left(\Phi_{2}^{\dagger} \Phi_{2}\right)-\left[\mu_{12}^{2}\left(\Phi_{1}^{\dagger} \Phi_{2}\right)+h . c .\right], \\
U^{(4)}=\lambda_{1}\left(\Phi_{1}^{\dagger} \Phi_{1}\right)^{2}+\lambda_{2}\left(\Phi_{2}^{\dagger} \Phi_{2}\right)^{2}+\lambda_{3}\left(\Phi_{1}^{\dagger} \Phi_{1}\right)\left(\Phi_{2}^{\dagger} \Phi_{2}\right)+\lambda_{4}\left(\Phi_{1}^{\dagger} \Phi_{2}\right)\left(\Phi_{2}^{\dagger} \Phi_{1}\right) \\
+\left[\lambda_{5} / 2\left(\Phi_{1}^{\dagger} \Phi_{2}\right)\left(\Phi_{1}^{\dagger} \Phi_{2}\right)+\lambda_{6}\left(\Phi_{1}^{\dagger} \Phi_{1}\right)\left(\Phi_{1}^{\dagger} \Phi_{2}\right)+\lambda_{7}\left(\Phi_{2}^{\dagger} \Phi_{2}\right)\left(\Phi_{1}^{\dagger} \Phi_{2}\right)+h . c .\right], \\
U^{(6)}=\kappa_{1}\left(\Phi_{1}^{\dagger} \Phi_{1}\right)^{3}+\kappa_{2}\left(\Phi_{2}^{\dagger} \Phi_{2}\right)^{3}+\kappa_{3}\left(\Phi_{1}^{\dagger} \Phi_{1}\right)^{2}\left(\Phi_{2}^{\dagger} \Phi_{2}\right)+\kappa_{4}\left(\Phi_{1}^{\dagger} \Phi_{1}\right)\left(\Phi_{2}^{\dagger} \Phi_{2}\right)^{2} \\
+\kappa_{5}\left(\Phi_{1}^{\dagger} \Phi_{1}\right)\left(\Phi_{1}^{\dagger} \Phi_{2}\right)\left(\Phi_{2}^{\dagger} \Phi_{1}\right)+\kappa_{6}\left(\Phi_{1}^{\dagger} \Phi_{2}\right)\left(\Phi_{2}^{\dagger} \Phi_{1}\right)\left(\Phi_{2}^{\dagger} \Phi_{2}\right) \\
+\kappa_{7}\left(\Phi_{1}^{\dagger} \Phi_{2}\right)^{3}+\kappa_{8}\left(\Phi_{1}^{\dagger} \Phi_{1}\right)^{2}\left(\Phi_{1}^{\dagger} \Phi_{2}\right)+\kappa_{9}\left(\Phi_{1}^{\dagger} \Phi_{1}\right)\left(\Phi_{1}^{\dagger} \Phi_{2}\right)^{2} \\
+\kappa_{10}\left(\Phi_{1}^{\dagger} \Phi_{2}\right)^{2}\left(\Phi_{2}^{\dagger} \Phi_{2}\right)+\kappa_{11}\left(\Phi_{1}^{\dagger} \Phi_{2}\right)^{2}\left(\Phi_{2}^{\dagger} \Phi_{1}\right)+\kappa_{12}\left(\Phi_{1}^{\dagger} \Phi_{2}\right)\left(\Phi_{2}^{\dagger} \Phi_{2}\right)^{2} \\
\left.+\kappa_{13}\left(\Phi_{1}^{\dagger} \Phi_{1}\right)\left(\Phi_{1}^{\dagger} \Phi_{2}\right)\left(\Phi_{2}^{\dagger} \Phi_{2}\right)+h . c .\right],
\end{gathered}
$$

where $\Phi_{i}$ are the Higgs doublets $\left[\Phi_{i}^{T}=\left(-i \omega_{i}^{+}, 1 / 2\left(v_{i}+\eta_{i}+i \chi_{i}\right)\right), i=1,2,\right], v_{1,2}$ are their vev's $\left[\left(v_{1}^{2}+v_{2}^{2}\right)^{1 / 2}=246 \mathrm{GeV}, v_{2} / v_{1}=\tan \beta\right]$. Radiative corrections to the $U_{\text {eff }}=U^{(2)}+U^{(4)}$ have been analyzed in [16-24]. The term $U^{(6)}$ have been considered in [8] where the one-loop threshold corrections to parameters $\kappa_{i}$ were obtained by using the effective potential method ${ }^{2}$. After transformation to the mass basis, the potential (1) has the form

$$
U_{\mathrm{eff}}=\frac{m_{h}^{2}}{2} h^{2}+\frac{m_{H}^{2}}{2} H^{2}+\frac{m_{A}^{2}}{2} A^{2}+m_{H^{ \pm}}^{2} H^{+} H^{-}+I_{3}+I_{4}+I_{5}+I_{6}+\ldots
$$

where $m_{h, H, A, H^{ \pm}}$are Higgs boson masses, $I_{3, \ldots 6}$ denote the interaction terms of physical scalars. The mixing angle $\alpha$ of the CP-even Higgs sector is specified by

$$
\tan 2 \alpha=\frac{\left(m_{Z}^{2}+m_{A}^{2}\right) s_{2 \beta}-2 \Delta \mathcal{M}_{12}^{2}}{\left(m_{A}^{2}-m_{Z}^{2}\right) c_{2 \beta}-\Delta \mathcal{M}_{11}^{2}+\Delta \mathcal{M}_{22}^{2}},
$$

where $\Delta \mathcal{M}_{i j}$ are radiative corrections to the mass matrix ${ }^{3}, s_{2 \beta}=\sin 2 \beta, c_{2 \beta}=\cos 2 \beta$, etc.

This approximate model must satisfy the following requirements.

\section{Existence of the Higgs mass basis}

In the general case, radiative corrections to the Higgs sector may lead to spontaneous symmetry breakdown [25] when the local minimum of the Higgs potential can degenerate or even disappear. Such a situation might take place in calculations of loop diagrams when obtained radiative corrections and Higgs masses might not correspond to eigenvalues of mass matrices containing all factors in front of the lagrangian terms of dimension two in terms of $S U(2)$ fields (as treated in effective field theory (EFT) approach). Under $\kappa_{i}=0$ assumption, one can check the correspondence using the transformation (31)-(35), (40) in [22] which is valid for a general THDM regardless of the method of $\lambda_{1, \ldots 7}$ calculations. The mass basis is automatically ensured in the EFT approach with or without dimension-six operators taken into account.

\footnotetext{
${ }^{2}$ One-loop threshold radiative corrections to $\kappa_{i}$ have been obtained under the assumption that all supersymmetric (SUSY) partners are heavy enough to be integrated out when the third generation of squarks has a common mass scale $M_{S}$.

${ }^{3}$ The explicit form of $\Delta \mathcal{M}_{i j}$ as well as the Higgs boson masses depending on parameters $\lambda_{i}$ and $\kappa_{j}$ can be found in [8].
} 


\section{Alignment limit}

The current Higgs couplings measurement at the LHC [26, 27] does not contradict the SM predictions, $g_{h u u} \approx g_{h d d} \approx g_{h V V} \approx 1$, where $u, d$ are up- and down-quarks, $V$ denotes gauge bosons. From the expressions $g_{h u u}=\cos \alpha / \sin \beta, g_{h d d}=-\sin \alpha / \cos \beta, g_{h V V}=\sin (\beta-\alpha)$ one can find an alignment condition [28, 29]

$$
\beta-\alpha \approx \pi / 2
$$

where $\alpha$ is defined by eq. (6).

\section{Perturbative unitarity}

The standard procedure to probe unitarity is to calculate two-particle scattering processes at tree level. All coupled channels for all particles should be considered, so the full treelevel analysis can be rather cumbersome. It was shown by Lee, Quigg and Thacker that at a very large scattering energy in the 't Hooft-Feynman gauge one can consider an amplitude for the scalar field theory instead of an amplitude for scattering of longitudinal intermediate bosons and physical Higgs particles with the accuracy of $O\left(M_{i} / \sqrt{s}\right)$ (the Goldstone boson equivalence theorem) [30]. In other words, all diagrams with propagators can be neglected and only vertex type interactions should be analyzed. This simplification is usually used in the literature [31, 32], however, as was noted in [33] scattering may never be in the regime with sufficiently large energies when particle masses in the propagators could be neglected. In this case, cubic couplings of large scalars must be taken into account which significantly modify the case.

For $S_{1} S_{2} \rightarrow S_{3} S_{4}$ scattering processes of scalars at finite $\sqrt{s}$ the perturbative unitarity constraints for the eigenvalues of $S$-wave partial amplitude matrix $a_{0}$ are [33]

$$
\begin{gathered}
\operatorname{Re}\left(a_{0}^{i}\right) \leq \frac{1}{2} \quad \forall i, \\
a_{0}=-\frac{2^{-\frac{1}{2}\left(\delta_{12}+\delta_{34}\right)}}{16 \pi}\left\{\left[\lambda\left(s, m_{1}^{2}, m_{2}^{2}\right) \lambda\left(s, m_{3}^{2}, m_{4}^{2}\right)\right]^{\frac{1}{4}}\left[\lambda^{1234}+\kappa^{125} \kappa^{345} \frac{1}{s-m_{5}^{2}}\right]\right. \\
\left.-\kappa^{135} \kappa^{245} f_{t}\left(s, m_{1, . .5}^{2}\right)-\kappa^{145} \kappa^{235} f_{u}\left(s, m_{1, \ldots 5}^{2}\right)\right\},
\end{gathered}
$$

where the factor $\delta_{12}\left(\delta_{34}\right)$ is 1 if particles $\{1,2\}(\{3,4\})$ are identical, and zero otherwise, $s=\left(p_{1}+p_{2}\right)^{2}$ is the Mandelstam variable, $m_{5}$ is the particle mass in a propogator, $\lambda^{1234}$ and $\kappa^{i j k}$ are quartic and trilinear couplings of scalars,

$$
\begin{aligned}
f_{t}\left(s, m_{1, \ldots 5}^{2}\right) & \equiv \frac{1}{s} \frac{1}{\left[\lambda\left(s, m_{1}^{2}, m_{2}^{2}\right) \lambda\left(s, m_{3}^{2}, m_{4}^{2}\right)\right]^{\frac{1}{4}}} \log \left(\frac{m_{1}^{2}+m_{3}^{2}-m_{5}^{2}-2 E_{1} E_{3}+2\left|\mathbf{p}_{1}\right|\left|\mathbf{p}_{3}\right|}{m_{1}^{2}+m_{3}^{2}-m_{5}^{2}-2 E_{1} E_{3}-2\left|\mathbf{p}_{1}\right|\left|\mathbf{p}_{3}\right|}\right), \\
f_{u}\left(s, m_{1, \ldots 5}^{2}\right) & \equiv f_{t}\left(s, m_{1}^{2}, m_{2}^{2}, m_{4}^{2}, m_{3}^{2}, m_{5}^{2}\right), \\
\lambda\left(s, m_{i}^{2}, m_{j}^{2}\right) & \equiv \frac{1}{s^{2}}\left(s^{2}+m_{i}^{4}+m_{j}^{4}-2 m_{i}^{2} m_{j}^{2}-2 s m_{i}^{2}-2 s m_{j}^{2}\right), \\
\left|\mathbf{p}_{1}\right| & =\frac{1}{2} \sqrt{s \lambda\left(s, m_{1}^{2}, m_{2}^{2}\right)}, \quad\left|\mathbf{p}_{3}\right|=\frac{1}{2} \sqrt{s \lambda\left(s, m_{3}^{2}, m_{4}^{2}\right)}
\end{aligned}
$$




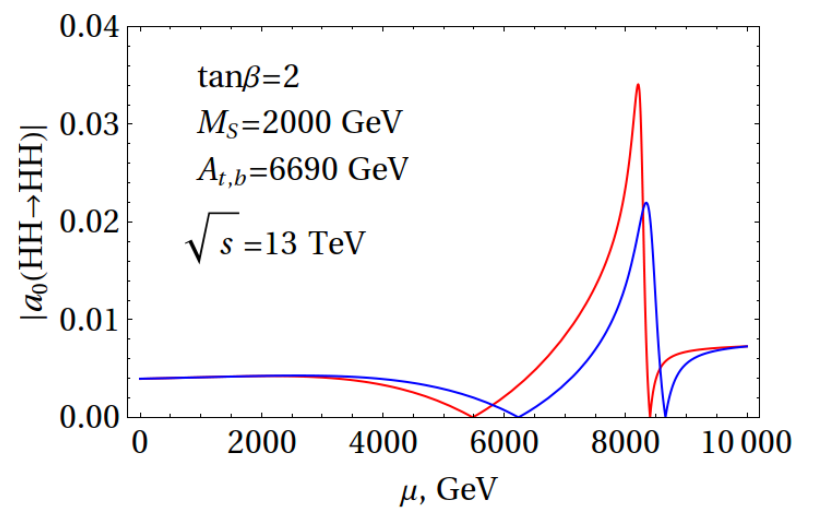

Figure 1. An absolute value of the zeroth partial wave of heavy CP-even Higgs bosons scattering $\mathrm{HH} \rightarrow \mathrm{HH}$ as a function of mass parameter $\mu$ for two approximations of the Higgs potential $U_{\text {eff }}$ decomposition : $U^{(2)}+U^{(4)}$ (blue line) and $U^{(2)}+U^{(4)}+U^{(6)}$ (red line) [see eq. (1)]

are the centre of mass three-momenta,

$$
E_{1}=\frac{s+m_{1}^{2}-m_{2}^{2}}{2 \sqrt{s}}, \quad E_{3}=\frac{s+m_{3}^{2}-m_{4}^{2}}{2 \sqrt{s}}
$$

are the energies of the particles in the center of mass frame.

It is obvious that unitarity constraints of the MSSM extension with dimension-six operators are different from ones with only renormalizable operators. Effective parameters $\kappa_{i}$ modify the Higgs masses and couplings [8]. An example of a constraint for heavy CP-even Higgs bosons scattering $\left|a_{0}(H H \rightarrow H H)\right|$ as a function of a Higgs superfield mass parameter $\mu$ at $\tan \beta=2, M_{S}=2 \mathrm{TeV}, A_{t, b}=6690 \mathrm{GeV}, \sqrt{s}=13 \mathrm{TeV}$ is presented in figure 1 . The red (blue) line corresponds to representation of the effective Higgs potential up to dimension-six (dimension-four) operators. As one can see the restrictions vary greatly in the range $\mu>3$ $\mathrm{TeV}$.

\section{Electroweak vacuum stability}

The MSSM vacuum stability bounds are constructed under the assumption that among nonzero vacuum expectation values of SUSY fields the deepest minimum of the effective potential coincides with the electroweak (EW) minimum [34]. The case of stability of the local minimum of the Higgs potential with dimension-six operators [see eq. (1)] was considered in [35] where it was shown that for the majority of the MSSM benchmark scenarios [36, 37] the allowed parameter regions coincide, but at the values of soft-SUSY breaking parameters of $\sim 5-10 \mathrm{TeV}$ the parameter space is stronger restricted compared to four-dim operators decomposition. As a rule, perturbative unitarity constraints are weaker than the vacuum stability bounds [38].

\section{The model features with a $28 \mathrm{GeV}$ pseudoscalar}

Following the MSSM approximation of [8] the considered model in the CP-conserving limit has the following free parameters: $\tan \beta$, pseudoscalar mass $m_{A}$, a SUSY scale $M_{S}$, Higgs superfield mass parameter $\mu$ and trilinear couplings of the third generation of squarks $A_{t}, A_{b}$, where for simplicity $A_{t}=A_{b}$. Assuming $m_{A}=28 \mathrm{GeV}, M_{S}=\{600,1000,2000,3500,5000\}$ $\mathrm{GeV}, \tan \beta=\{1,2,3,5,15,20\}$, one can adjust parameters $\mu, A_{t, b}$ in such a way that a light CPeven Higgs boson $h$ has the mass $125 \mathrm{GeV}$ at the alignment limit (7). This leads to stringent 
Table 1. Benchmark points under the assumption that $m_{A}=28 \mathrm{GeV}, m_{h}=125 \mathrm{GeV}$ in the alignment limit

\begin{tabular}{lllll}
\hline BP & $\tan \beta$ & $M_{S}(\mathrm{GeV})$ & $A_{t, b}(\mathrm{GeV})$ & $\mu(\mathrm{GeV})$ \\
\hline 1 & 2 & 2000 & 8800 & 5320 \\
2 & 3 & 2000 & 7820 & 6450 \\
3 & 5 & 1000 & 3385 & 5040 \\
4 & 5 & 2000 & 6690 & 7960 \\
\hline
\end{tabular}

Table 2. Masses of Higgs bosons, couplings and partial wave values for the process $H H \rightarrow H H$ under the assumption $m_{A}=28 \mathrm{GeV}, m_{h}=125 \mathrm{GeV}$

\begin{tabular}{llllllll}
\hline $\mathrm{BP}$ & $m_{H}$ & $m_{H^{ \pm}}$ & $g_{\text {huu }}$ & $g_{h d d}$ & $g_{h V V}$ & \multicolumn{2}{c}{$\operatorname{Re}\left[a_{0}(H H \rightarrow H H)\right]$} \\
& $(\mathrm{GeV})$ & $(\mathrm{GeV})$ & & & & $\sqrt{s}=8 \mathrm{TeV}$ & $\sqrt{s}=13 \mathrm{TeV}$ \\
\hline 1 & 134.4 & 129.7 & 1.1 & 0.7 & 1.0 & -0.0175 & -0.0174 \\
2 & 132.3 & 130.0 & 1.0 & 0.9 & 1.0 & -0.0182 & -0.0181 \\
3 & 127.7 & 127.3 & 1.0 & 1.0 & 1.0 & -0.1376 & -0.1369 \\
4 & 130.4 & 131.3 & 1.0 & 1.0 & 1.0 & -0.0222 & -0.0221 \\
\hline
\end{tabular}

constraints on the parameter space: for $\tan \beta$ and $M_{S}$ under consideration only four parameter sets (benchmark points, BP's) exist, see table 1.

Note that the condition $m_{A}=28 \mathrm{GeV}$ combined with $h(125 \mathrm{GeV})$ in the alignment limit is possible only for Higgs potential decomposition up to dimension-six operators with large values of $A_{t, b}, \mu$ that is rather unusual for common LHC scenarios [36, 37] where $A_{t, b}, \mu \sim O$ $(100 \mathrm{GeV})$. Nevertheless, for these BP's the Higgs potential minimum is stable, perturbative unitarity conditions for the probing scattering process $H H \rightarrow H H$ are also satisfied (see table 2 and figure 1) and the values of radiative corrections to $\lambda_{i}$ and $\kappa_{j}$ are below 1 , as shown in figure 2 for the BP1. One can see from table 2 that the Higgs sector is nearly degenerate with masses at the electroweak scale.
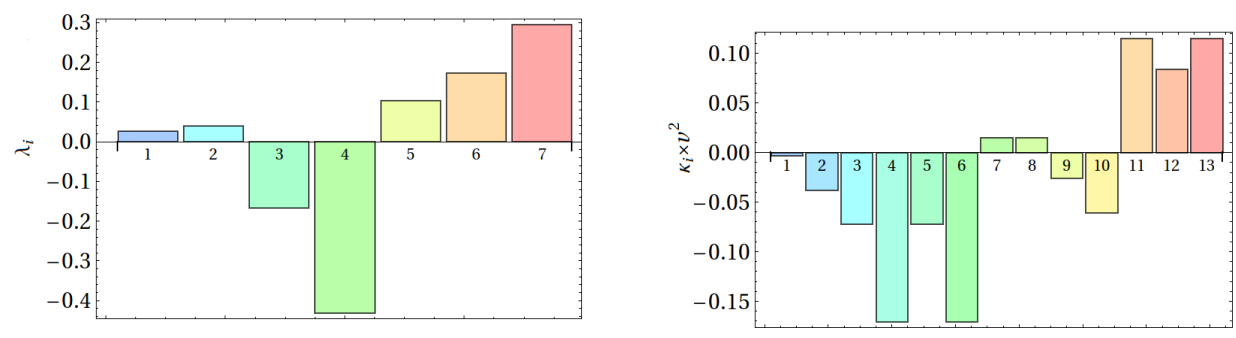

Figure 2. Self-couplings $\lambda_{i}$ (left) and $\kappa_{j}$ (right) of the Higgs potential (1)-(4) calculated for the BP1

\subsection{Cross section of the process $g g \rightarrow \mu^{+} \mu^{-} b \bar{b}$}

As was reported by the CMS Collaboration [5] an excess of the muon pair production in the mass range $12-70 \mathrm{GeV}$ at about $28 \mathrm{GeV}$ in association with a $b$-quark jet and an additional jet has been observed in two event categories denoted in the following by SR 1 and SR 2 at $\sqrt{s}=8$ and $13 \mathrm{TeV}$. Reconstructed cross sections of the excess are $4.1 \pm 1.4 \mathrm{fb}$ (SR1) and $4.2 \pm 1.7$ $\mathrm{fb}(\mathrm{SR} 2)$ for $\sqrt{s}=8 \mathrm{TeV}$ and $1.4 \pm 0.9 \mathrm{fb}(\mathrm{SR} 1)$ and $-1.5 \pm 1.0 \mathrm{fb}(\mathrm{SR} 2)$ for $\sqrt{s}=13 \mathrm{TeV}$. Assuming that this excess is a light pseudoscalar one can evaluate production cross section at 
complete tree level for the process $g g \rightarrow A b \bar{b} \rightarrow \mu^{+} \mu^{-} b \bar{b}$, see the signal diagrams in figure 3 . Corresponding to the CMS event selection [5]
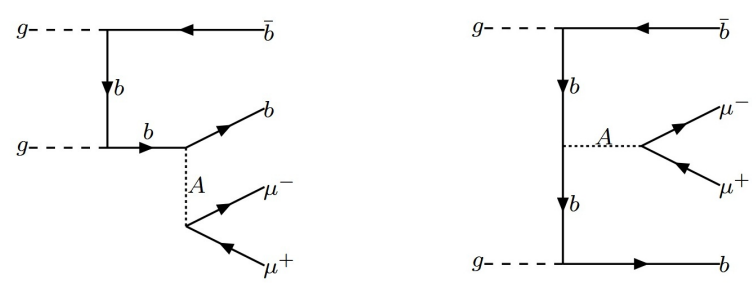

Figure 3. Feynman diagrams with production and decay of a CP-odd Higgs boson $A$ associated with b-quarks

$$
\begin{array}{rll}
\text { Muons : } & p_{T}>25 \mathrm{GeV}, & |\eta|<2.1, \quad m_{\mu^{+} \mu^{-}}>12 \mathrm{GeV}, \\
b: & p_{T}>30 \mathrm{GeV}, & |\eta| \leq 2.4, \\
\bar{b}: & p_{T}>30 \mathrm{GeV}, & 2.4 \leq|\eta| \leq 4.7 \quad(\text { SR1) } \quad|\eta| \leq 2.4 \quad \text { (SR2), }
\end{array}
$$

the following cuts have been imposed

cut A : all irreducible background diagrams with intermediate photons and gauge bosons are omitted, phase space cuts are imposed for SR1 event category;

cut B : all irreducible background diagrams with intermediate photons and gauge bosons are omitted, phase space cuts are imposed for SR2 event category, $25 \mathrm{GeV} \leq m_{\mu^{+} \mu^{-}} \leq 32$ $\mathrm{GeV}$;

cut C : complete tree level set of diagrams is calculated, phase space cuts are imposed for SR1 event category;

cut D : complete tree level set of diagrams is calculated, phase space cuts are imposed for SR2 event category.

Cross sections evaluated by CompHEP [39] package are presented in table 3. As expected, cross sections for complete sets of diagrams using phase space cuts A and B coincide with the production $\times$ decay approximation $\sigma(g g \rightarrow b \bar{b} A) \mathrm{BR}\left(A \rightarrow \mu^{+} \mu^{-}\right)$. One can see that the evaluated cross sections are smaller than the reconstructed cross sections of the CMS excess.

\subsection{Top quark decay}

The most sensitive way to search for exotic processes is to analyze the interactions with top quarks due to the large top quark Yukawa coupling. As was demonstrated above in the case of light pseudoscalar, the masses of Higgs bosons are of the order of EW scale. For all BP's $m_{H^{ \pm}}<m_{\text {top }}$ (see table 2), so in this case the exotic decay of the top quark $t \rightarrow H^{ \pm} b$ should take place. The experimental constraints for this process are the following:

(i) for $\tan \beta<5$ the ATLAS upper limit of $B R\left(t \rightarrow H^{+} b\right)$ is $1-5 \%$ (95\% CL) in the assumption that the dominant hadronic decay mode is $H^{+} \rightarrow c \bar{s}$ [40];

(ii) for $\tan \beta \geq 5$ the ATLAS and CMS upper limits on $B R\left(t \rightarrow H^{+} b\right) B R\left(H^{+} \rightarrow \tau^{+} v_{\tau}\right)$ are $1.3-0.2 \%$ [40] and $1.2-0.5 \%$ [41]. 
Table 3. Cross section of the process $g g \rightarrow \mu^{+} \mu^{-} b \bar{b}$ in $\mathrm{fb}$

\begin{tabular}{llllll}
\hline & & \multicolumn{2}{c}{ SR1 } & \multicolumn{2}{c}{ SR2 } \\
$\sqrt{s}$ & BP & cut A & cut C & cut B & cut D \\
\hline \multirow{3}{*}{$8 \mathrm{TeV}$} & 1 & 0.009 & 10.094 & 0.065 & 267.240 \\
& 2 & 0.020 & 13.242 & 0.134 & 236.750 \\
& 3 & 0.056 & 8.814 & 0.384 & 270.810 \\
& 4 & 0.057 & 9.800 & 0.387 & 223.870 \\
& & & & & \\
$13 \mathrm{TeV}$ & 1 & 0.027 & 55.994 & 0.148 & 571.790 \\
& 3 & 0.058 & 48.692 & 0.310 & 609.650 \\
& 4 & 0.165 & 53.642 & 0.902 & 610.500 \\
& 4 & 0.191 & 31.760 & 0.905 & 587.320 \\
\hline
\end{tabular}

In the model under consideration the main decay of charged Higgs boson is to pseudoscalar $A$ and $W^{+}$boson (90-99\%), and therefore comparison for $\tan \beta<5$ is rather ambiguous. Nevertheless, numerical CompHEP evaluations yield $B R\left(t \rightarrow H^{+} b\right)=5.4 \%$ (2.6\%) for BP1 (BP2). For $\tan \beta=5$ the value of $B R\left(t \rightarrow H^{+} b\right) B R\left(H^{+} \rightarrow \tau^{+} v_{\tau}\right)$ of about $0.13 \%$ (BP3) and $0.09 \%$ (BP4) which is in agreement with experimental data.

\section{Conclusion}

The scenarios with a light pseudoscalar Higgs boson of the MSSM extended by dimension-six operators in the Higgs sector have been analyzed. It was shown that the CP-odd Higgs boson with mass of $28 \mathrm{GeV}$ can be embedded in the model if the soft-SUSY breaking parameters are around 3-9 TeV and the SUSY mass scale $M_{S}$ is of a few TeV. Such an unusual parameter regime does not spoil the perturbative unitarity ${ }^{4}$ and vacuum stability of the model. In such scenarios, the mass spectrum of Higgs bosons is splitted weakly at the EW scale. The top quark decay to charged Higgs boson and bottom quark as a sensitive way for a new physics search has been found promising. It was shown that model predictions do not contradict the direct experimental data. At the same time, estimated cross sections $\sigma\left(g g \rightarrow \mu^{+} \mu^{-} b \bar{b}\right)$ are from several times to about an order of magnitude smaller than the cross sections experimenally reconstructed for the CMS excess.

The authors are grateful to H. Bahl, O. Kodolova, A. Nikitenko and G. Weiglein for useful discussions. This work was supported by the Russian Science Foundation Grant No. 16-12-10280.

\section{References}

[1] P. Ghosh, D. E. Lopez-Fogliani, V. A. Mitsou, C. Munoz and R. R. Austri, JHEP 11, 102 (2014)

[2] G. Branco et al., Phys. Rept. 516, 1 (2012) [arXiv: 1106.0034 [hep-ph]]

[3] U. Ellwanger et al., Phys. Rept. 496, 1 (2010) [arXiv: 0910.1785 [hep-ph]]

[4] F. Bezrukov, D. Gorbunov, I. Timiryasov, arXiv:1812.08088 [hep-ph]

[5] CMS Collaboration, JHEP 11, 161 (2018); 1711, 010 (2017)

[6] ATLAS Collaboration, ATLAS-CONF-2019-036, https://cds.cern.ch/record/2684853

\footnotetext{
${ }^{4}$ The estimation has been performed for a limited number of channels.
} 
[7] A. Heister, arXiv: 1610.06536 [hep-ex]

[8] M. N. Dubinin and E. Yu. Petrova, Phys. Rev. D 95, 055021 (2017) [arXiv:1612.03655 [hep-ph]]

[9] M. N. Dubinin and E. Yu. Petrova, Int. J. Mod. Phys. A 33, 1850150 (2018) [arXiv:1709.10301 [hep-ph]]

[10] C. Beskidt, W. de Boer, and D.I. Kazakov, Phys. Lett. B 782, 69 (2018)

[11] S. I. Godunov, V. A. Novikov, M. I. Vysotsky, and E. V. Zhemchugov, JETP Lett. 109, 358 (2019)

[12] ATLAS Collaboration, Phys. Lett. B 716, 1 (2012)

[13] CMS Collaboration, Phys. Lett. B 716, 30 (2012)

[14] ATLAS and CMS Collaborations, JHEP 1608, 045 (2016)

[15] ATLAS and CMS Collaborations, Phys. Rev. Lett. 114, 191803 (2015)

[16] S. Coleman and E. Weinberg, Phys. Rev. D 7, 1888 (1973)

[17] H. E. Haber and R. Hempfling, Phys. Rev. D 48, 4280 (1993)

[18] M. Carena, J. Ellis, A. Pilaftsis, and C. E. M. Wagner, Nucl. Phys. B 586, 92 (2000)

[19] S. Y. Choi, M. Drees, and J. S. Lee, Phys. Lett. B 481, 57 (2000)

[20] M. Carena, M. Quiros and C. E. M. Wagner, Nucl. Phys. B 461, 407 (1996)

[21] M. Carena, J. R. Espinosa, M. Quiros, and C. E. M. Wagner, Phys. Lett. B 355, 209 (1995)

[22] E. Akhmetzyanova, M. Dolgopolov, and M. Dubinin, Phys. Rev. D 71, 075008 (2005)

[23] E. Akhmetzyanova, M. Dolgopolov, and M. Dubinin, Phys. Part. Nucl. 37, 677 (2006)

[24] M. N. Dubinin and E. Yu. Petrova, Yad. Phys. 79, 302 (2016)

[25] H. D. Politzer, S. Wolfram, Phys. Lett. B 82, 242 (1979); 83, 421 Errata (1979)

[26] J. Brandstetter, arXiv:1801.07926v1 [hep-ex]

[27] M. Malberti, Nuovo Cimento C 40, 182 (2017)

[28] M. Carena, H. E. Haber, I. Low, N. R. Shah, and C. E. M. Wagner, Phys. Rev. D 91, 035003 (2015)

[29] D. Asner et al., ILC Higgs white paper (The Division of Particles and Fields of the American Physical Society, Minneapolis, 2013) 1 [arXiv:1310.0763 [hep-ph])]

[30] B. W. Lee, C. Quigg, and H. B. Thacker, Phys. Rev. D 16, 1519 (1977)

[31] A. G. Akeroyd, A. Arhrib, and E. Naimi, Phys. Lett. B 490, 119 (2000)

[32] S. Kanemura and K. Yagyu, Phys. Lett. B 751, 289 (2015)

[33] M. D. Goodsell, F. Staub, Eur. Phys. J. C 78, 649 (2018)

[34] W. G. Hollik, G. Weiglein, and J. Wittbrodt, JHEP 1903, 109 (2019)

[35] M. N. Dubinin and E. Yu. Petrova, EPJ Web Conf. 158, 02005 (2017)

[36] M. Carena, S. Heinemeyer, O. Stal, C. E. M. Wagner, and G. Weiglein, Eur. Phys. J. C 73, 2552 (2013)

[37] H. Bahl, S. Liebler, T. Stefaniak, Eur.Phys.J. C 79, 279 (2019) 279

[38] F. Staub, Phys. Lett. B 789, 2013 (2019).

[39] CompHEP Collaboration, Nucl. Instrum. Methods A534, 250 (2004); A. Pukhov et al., arXiv:hep-ph/9908288

[40] ATLAS Collaboration, JHEP 03, 088 (2015); Eur. Phys. J. C 73, 2465 (2013)

[41] CMS Collaboration, JHEP 1511, 018 (2015) 